\title{
Aproximación a una metodología de análisis de las emociones en el discurso político televisado
}

\author{
Sandra Méndez-Muros ${ }^{1}$ \\ ${ }^{1}$ Departamento de Periodismo II Universidad de Sevilla, España | sanmenmur@us.es | \\ https://orcid.org/0000-0003-4312-9798
}

\begin{abstract}
Resumen: En el contexto de investigaciones recientes desde las neurociencias, las cuales presentan ciertas limitaciones para el análisis científico, nos proponemos descubrir una metodología de análisis de las emociones en el discurso político televisado. En primer lugar, revisamos la literatura sobre los métodos y técnicas de investigación empleados actualmente en los trabajos sobre el papel de las emociones en el discurso político en general y, particularmente, en los medios de comunicación. En segundo lugar, planteamos un estudio descriptivo y de análisis de contenido cualitativo siguiendo los principios de inferencia emocional, estrategias y disparadores lingüísticos propuestos por Ungerer. La muestra de análisis está compuesta por las 13 comparecencias de los líderes de los cinco principales partidos políticos tras conocerse los resultados de las elecciones autonómicas andaluzas el 2 de diciembre de 2018 en el especial informativo de la primera cadena de Televisión Española. Entre las principales conclusiones se encuentran que en el análisis de las emociones en el discurso político se dan variados métodos sin una clara tendencia, así como la utilidad del método empleado en su aplicación al mensaje televisado, dada la observación de un amplio uso de estrategias emocionales en los discursos de los líderes políticos.
\end{abstract}

Palabras clave: Emoción; Neurociencia; Discurso Político; Metodologías; Televisión.

Approach to an Emotion Analysis Methodology in Televised Political Speech

\begin{abstract}
In the context of recent research from neurosciences, which present certain limitations for scientific analysis, we propose to discover know a methodology for the analysis of emotions in televised political speech. Firstly, we review the literature on the research methods and techniques currently used in the works on the role of emotions in political discourse in general and, particularly, in the media. Secondly, we propose a descriptive study and qualitative content analysis following the principles of emotional inference, strategies and language triggers proposed by Ungerer. The analysis sample comprises the 13 appearances of the leaders of the five main political parties after knowing the results of the Andalusian regional elections in the special news of the Television Española channel 1 on December 2, 2018. Among the main conclusions, we can assert that in the analysis of emotions in political speech there are various methods without a clear trend, as well as the usefulness of the method used in its application to the televised message, given the observation of a wide use of emotional strategies in the speeches of political leaders.
\end{abstract}

Keywords: Emotion; Neuroscience; Political Speech; Methodologies; Television.

\section{Introducción}

La razón y la emoción cumplen un papel importante en la toma de decisiones del ser humano. El $90 \%$ de las decisiones se toman de manera inconsciente guiadas por las emociones, que son las que movilizan constantemente a la razón, y el $80 \%$ de información subconsciente está relacionada con las decisiones libres que tomamos en nuestra vida diaria (Gutiérrez-Rubí, 2009: 85). El mecanismo más influyente en la toma de decisiones es la empatía -proceso de "ponerse en lugar de"-, por lo que para que alguien se incline por un producto, una idea, un programa político, etc. es necesario activar aquellas emociones que le impulsan a tomar una u otra decisión.

La tecnología ha permitido descubrir en las últimas décadas más sobre el funcionamiento del cerebro que en toda la historia de la humanidad. El registro y control cerebrales a través de la tecnología comporta un elevado dominio de la toma de decisiones humanas y la construcción de perfiles concretos (intereses, valores, gustos, preferencias). 
En este contexto, las neurociencias intentan explicar cómo funciona el cerebro y sus relaciones con la conciencia, valiéndose fundamentalmente del método de observación bajo la premisa de que, si el cerebro es la base del comportamiento humano, su estudio nos dará luz sobre distintas materias. Aunque el futuro apunta hacia la conexión total entre el cerebro humano y el ordenador, en la actualidad sólo se puede conocer el cerebro más allá de la observación del comportamiento y, por ende, la motivación de la toma de decisiones a través de dos vías: a) el comportamiento digital mediante las técnicas de neuroinformación o procesamiento de macrodatos y b) las técnicas de neurotecnología que estudian las bases biológicas de la conducta humana a través del análisis del funcionamiento del sistema nervioso.

La primera vía sienta sus bases en que los usuarios de tecnologías dejan un rastro en la red en forma de datos, lo que permite hacer retratos gracias a algoritmos que originan unos patrones. Los macrodatos o Big data capacitan la visualización, recuperación y gestión de grandes volúmenes de datos aplicando la minería de datos u obtención de modelos de predicción como los árboles de decisión o las redes neuronales artificiales, que son entrenadas con el fin de construir un modelo que reproduce el método de aprendizaje del cerebro humano y realiza predicciones mediante algoritmos de patrones de comportamiento y acción (Tallón-Ballesteros, 2019). Esto permite controlar el itinerario de un usuario y parametrizar las emociones o la empatía que produce un producto o una idea en la red. En cuanto a la segunda vía, algunas técnicas como la biorretroalimentación, el reconocimiento facial, el rastreo ocular (eye tracking) o la resonancia magnética funcional, entre otras, analizan mediante aparatos de medición de la actividad cerebral las reacciones que se producen de manera inconsciente en las personas al ser estimuladas con el diseño, la imagen, el discurso, el video, la web, etc., que se desea evaluar.

Aparte de las tradicionales encuestas, ambos campos son explorados por los asesores políticos, uno de los grupos con mayor interés por conocer el mecanismo cerebral en la toma de decisiones, especialmente en período electoral. En el ámbito político español ya se están dando los primeros pasos en el uso de la neuroinformación. El 6 de diciembre de 2018 entró en vigor la nueva Ley Orgánica de Protección de Datos Personales y Garantía de los Derechos Digitales (LOPDGDD), que permite a los partidos políticos españoles "utilizar datos personales obtenidos en páginas web y otras fuentes de acceso público para la realización de actividades políticas durante el periodo electoral". Empresas a sueldo de partidos políticos pueden espiar en la red para realizar perfiles ideológicos de los ciudadanos de cara a enviarles propaganda ideológica a medida. Igualmente, en las campañas electorales de México, Estados Unidos o Rusia se han llevado a cabo experimentos que miden las reacciones cerebrales ante determinados líderes o programas políticos, desarrollando todo un campo de conocimiento sobre el votante en la denominada neuropolítica.

En este sentido, el neuromarketing y la neurocomunicación son empleados por los equipos de asesores que diseñan y cuidan la imagen de los líderes políticos. Las técnicas de neurocomunicación permiten conocer los mecanismos emocionales que están detrás de las reacciones ante determinados mensajes informativos e implementar estrategias de diseño de contenidos informativos, lo que ha supuesto que de nuevo vire el papel de los medios respecto al público. Al principio, el mensaje se daba de tú a tú, después el mensaje se dirigía a la masa y de nuevo se busca ofrecer mensajes individualizados porque se entiende que tienen más impacto cuando se personaliza y se adecua a los gustos y necesidades de cada uno.

No obstante, la aplicación de la neuroinformación como método de análisis por el requerimiento de test encargados de validar la calidad y veracidad de los datos de partida, así como el almacenamiento y el tiempo generado para descubrir las relaciones de causa-efecto, restringe la fuente disponible de datos. Otra limitación es la necesidad de que el sistema de normativas y regulaciones sobre seguridad y privacidad sea estable para el uso de algoritmos. 
Por su parte, la neurotecnología presenta un elevado coste económico y de recursos humanos y gran especificidad del sistema tecnológico, aparte de que las muestras son reducidas y poco significativas, así como la imposibilidad de análisis comparativos y las garantías ofrecidas por la tecnología empleada en el proceso de investigación plantean resultados cuestionables (Gómez \& Bandrés, 2014: 410-411). Asimismo, la mera aplicación de un programa estadístico sobre los resultados de la aplicación de técnicas neurológicas conlleva la aceptación de unos datos que apuntan más a estudios de mercado que al producto de una investigación científica.

Por todo ello, exponemos, en primer lugar, los métodos empleados en investigaciones recientes sobre el análisis científico de las emociones en el discurso político aparte de las neurociencias y, específicamente, en los medios de comunicación, para en, segunda instancia, proponer un método de análisis en el medio televisivo tan complejo, donde el mensaje es "un sistema de signos que conducen al estudio de la perspectiva teórica analítica” (Cebrián, 1983: 35).

\section{Métodos Actuales para el Estudio del Discurso Político Emocional}

Aparte de las neurociencias, las emociones han sido abordadas desde la psicología, las teorías de la comunicación y la pragmática, la oratoria, el discurso, la argumentación, la inteligencia emocional, la educación, la inteligencia social, el racionalismo y la cognición, la semiótica, la lingüística (actos de habla, juegos, recursos estilísticos), el lenguaje no verbal, etc., pero especialmente, han sido la oratoria -desde el pathos en la retórica aristotélica-, la propaganda, la persuasión y la manipulación los conceptos a los que están vinculadas especialmente en multitud de trabajos científicos, sobre todo aquéllos que versan sobre el discurso político.

Su relación con la persuasión y la manipulación la ha llevado a ser considerada tradicionalmente como origen de falacias (argumentum ad populum o argumentum ad passiones) (Nocetti, 1990) al miedo, a la compasión, a las consecuencias, al halago, a la culpa, al ridículo, al victimismo, etc., si bien Medina (2015: 124) defiende que "recurrir a la emoción en la argumentación no implica necesariamente que el argumento que se está tratando sea falaz, puesto que al ser examinado con detenimiento puede ser razonable y apropiado". Conjuntamente, su relación con la propaganda política ha sido ampliamente estudiada, dado que "existen en la psiquis de los pueblos sentimientos conscientes o inconscientes que la propaganda capta y explota" (Domenach, 1968).

Esto es especialmente relevante en el estudio del papel de los medios de comunicación, donde se contempla como una carga perjudicial: "la óptica propagandística dota al periodismo de una apariencia negativa cuando interviene la emoción. Los recursos emocionales impiden el conocimiento, la verdad y la razón, además de dificultar la toma de decisiones y de competir con las máximas de la objetividad y la verdad" (Méndez, 2014: 96).

En los estudios más recientes sobre el discurso político emocional se encuentran diferentes propuestas metodológicas, algunas de las cuales incluyen las neurociencias, mediante el compendio de obras más relevantes sobre el papel de las emociones y concretamente del neuromarketing (Maneiro, 2017), la revisión documental de diferentes postulaciones que relacionan la neurociencia con el comportamiento electoral (Arteaga, 2018) o el experimento con actividad electrodérmica para medir la atención y emoción en un grupo de personas sobre los discursos de varios líderes políticos empleando técnicas estadísticas con modelos para estudios longitudinales sobre los datos recogidos (Tapia, Matellanes, Berdón \& Martín, 2016). 
En otras investigaciones se recogen estrategias semióticas a nivel enunciativo, retórico, lingüístico, afectivo y gestual, que son reconocibles por el destinatario mediante inferencias abductivas (Eggs, 2008); la revisión documental para desarrollar un análisis de la lógica de vectores emocionales y su labor en ejes conceptuales y discursivos (Cossarini, 2019); la descripción del contexto institucional y las reglas del juego de una campaña electoral y la utilización de las emociones desde las intervenciones de políticos (Cardona y Londoño, 2018) y del contexto extralingüístico que configura y enmarca a los discursos políticos de una campaña electoral, siguiendo los ejes propuestos por Plantin (López, 2018); los repertorios emocionales (condiciones físicas; creencias metafísicas, religiosas y cosmológicas; usos y costumbres; diferencias lingüísticas y normas sociales) que distingue a la sociedad humana (Gil, 2016); el método de análisis multivariante que integra las emociones como variables en un modelo de regresión con los datos obtenidos de una encuesta postelectoral (González, 2019); la identificación y catalogación de los actos de habla según las emociones en el discurso político de mítines partiendo de la Teoría de la Valoración (Jiménez, 2017); la entrevista en profundidad donde se analizan estrategias de manejo emocional empleadas en la acción colectiva (Gravante \& Poma, 2018); el modelo basado en el pathos proyectivo en alocuciones orales monologales que considera diferentes niveles de organización en el plano lingüístico (matriz, estrato narrativo y estrato argumentativo) de las emociones (Dagatti, 2017); el análisis del discurso político para analizar las estrategias de varios candidatos electorales (Mendieta, 2018); o el enfoque historiográfico transversal de las emociones (Sierra, 2015).

Algunos estudios sobre el discurso político emocional en los medios de comunicación emplean técnicas propias del neuromarketing en los discursos políticos mediatizados (Gómez \& Bandrés, 2014), mientras que otros métodos contemplados son la descripción y la revisión documental para analizar las emociones humanas en la política electoral y partidista (Valdez, Huerta \& Díaz, 2014); el storytelling o arte de narrar una historia conectando con los receptores del mensaje como herramienta de análisis de las emociones (Cárdenas, 2013); la detección y clasificación de indicadores racionales y emocionales utilizados de forma habitual en comunicación política y especialmente en el mensaje audiovisual de campaña electoral (Espí, 2017); o el análisis del estilo narrativo y de las técnicas del relato en los discursos periodísticos del espacio político (Arroyas \& Pérez, 2009).

\section{Objetivo y Metodología}

En este trabajo nos proponemos conocer una metodología de análisis efectivo de las emociones en el discurso político televisado. Descartados los métodos basados en las neurociencias y considerando que "para suscitar la emoción, es indispensable la especificación" (Perelman \& Olbrechts-Tyteca, 1989: 238) y que "para aflorar las emociones deseadas en electorado o la ciudadanía, los medios más importantes son los visuales y los lingüísticos" (Jiménez, 2017), realizamos, en primer lugar, una investigación de la literatura sobre los métodos y técnicas de investigación empleados recientemente en los trabajos sobre el papel de las emociones en el discurso político en general y, particularmente, del discurso político en los medios de comunicación.

A continuación, planteamos un estudio descriptivo y de análisis de contenido cualitativo siguiendo los principios de inferencia emocional, estrategias y disparadores lingüísticos propuestos por Ungerer (1997: 314) sobre la base del sistema de implicaturas presentes en el intercambio de significados en el acto de comunicación. Estos principios son: 1) Principio de proximidad, el cual apela a la concentración de lo que es cercano al lector, mediante el empleo de ítems deícticos, términos de parentesco y formas cariñosas de dirigirse a los demás para dejar clara la posición de nosotros frente al resto. 2) Principio de animacidad, que invita a concentrarse en lo que pone en peligro la vida o lo que genera vida para los humanos; el vocabulario suele relacionarse con el desastre o la calamidad (asesinato, violación, asalto, temblor, bajas, víctimas, heridos). 3) Principio de rango y número, que llama a converger en lo que concierne a mucha gente y que es importante; se usan numerales y otras expresiones de cantidad y títulos. 
4) Principio de evaluación emocional, que sustenta estimaciones fundadas en las normas culturales y se sirve de adjetivos evaluativos e ítems lexicales con connotaciones positivas y negativas. 5) Principio de intensidad de la presentación, que recurre a ítems que proyectan el proceso de evaluación a través de detalles ingeniosos y enlaces metafóricos con dominios emocionalmente establecidos. 6) Principio del contenido emocional, que menciona aspectos emocionales de eventos de forma explícita, con descriptivos (adjetivos, sustantivos y verbos).

La novedad radica en que, en lugar de textos publicados en prensa para los que estos principios fueron propuestos, han sido aplicados a documentos audiovisuales orales, las 13 comparecencias de los líderes regionales y nacionales de los cinco principales partidos políticos tras conocerse los resultados de las elecciones autonómicas andaluzas el 2 de diciembre de 2018 en el especial informativo de la primera cadena de Televisión Española. Los discursos han sido transcritos para su posterior análisis de elementos exclusivamente lingüísticos con la atención de que las emociones se presentan "como efectos posibles que un determinado acto de lenguaje puede producir en una situación dada", puesto que "el lenguaje verbal, del lenguaje de la imagen o de otros medios de expresión como los gestos o las mímicas, el empleo de palabras o de rasgos icónicos no constituyen necesariamente la prueba de la existencia de una emoción" (Charaudeau, 2011: 110). En un paso más sobre la construcción del discurso, hemos de reparar en "la construcción de modelos mentales en la memoria episódica, que incluyen nuestras propias opiniones y emociones asociadas con un evento oído o leído" (Van Dijk, 2006: 58).

\section{Resultados}

\subsection{Principio de Proximidad}

Todos los líderes políticos evidencian la dicotomía nosotros versus ellos, siendo el principal eje temático el bloque de izquierda-derecha. Si bien no se emplean términos de parentesco ni fórmulas afectivas, queda patente la división ideológica por la recurrencia a lugares comunes a través de los deícticos, especialmente en los partidos de más reciente creación (Ciudadanos, Adelante Andalucía y Vox). En primer lugar, esto se demuestra en la autodefinición explícita de la mayoría de los partidos. El PP se presenta como la alternativa y el cambio ("representábamos precisamente esa garantía de cambio en Andalucía") y recuerda su espacio ideológico frente a otras fuerzas conservadoras ("el centro derecha moderado, reformista y liberal que encarna la mayor internacional política del mundo", "decirles a aquellos que han podido confiar en otras opciones políticas que no encontrarán al PP fuera del Partido Popular"). Adelante Andalucía se retrata como "la única fuerza política que ha sabido encarnar el espíritu del 4 de diciembre, el espíritu del 28 de febrero, el espíritu de la dignidad andaluza en forma de derechos sociales, justicia social y fraternidad", como "la única fuerza política que ha puesto la dignidad andaluza en el centro" y perfila su posición ("nos negamos a hacerles el favor a los poderosos de este país"). Y Vox se centra más en calificarse a sí mismo para aumentar su definición por ser novedoso en el espectro político ("nosotros somos un partido regenerador", "ahora es el momento de decir alto y fuerte quiénes somos").

La otra definición viene dada por los opositores políticos. Para el PSOE, es determinante la división entre los partidos democráticos y la extrema derecha representada por Vox. Esto lo lleva a definirlo como "partido que defiende la quiebra de la convivencia", con un "discurso xenófobo, que justifica la violencia hacia las mujeres y que justifica el enfrentamiento y la violencia entre los ciudadanos". EI PP, por su parte, carga contra la continuidad del PSOE ("ya se notaba un cansancio y un hastío por parte de la población", "lo que viene diciendo la mayoría de españoles en el rechazo de sus políticas sectarias e irresponsables [...], gracias a los apoyos de unos socios muy poco recomendables"). 
Ciudadanos hace una declaración de intenciones sobre su rechazo a la dicotomía ("yo no quiero una España de rojos y azules, quiero una España de ciudadanos libres e iguales"), pero ataca al PSOE de manera más clara ("vamos a intentar unir a los españoles", "los españoles le darán la espalda en las urnas y nos darán su apoyo", "a los que han estado durante cuarenta años viviendo de los andaluces, se les acabó el chollo"). También ataca al el PP ("a los que han llenado sus vitrinas de derrotas y que hoy han vuelto a perder") y a una oposición sin nombrar ("a algunos no les ha gustado que viniéramos mucho", "algunos nos decían que nos fuéramos a nuestra tierra"). Adelante Andalucía carga contra todos los opositores en su discurso: contra el PSOE y el PP ("los partidos viejos han tenido el peor resultado de toda su historia en Andalucía"), contra el PSOE ("frente a los que planteaban Andalucía como un segundo plato después de haber perdido unas primarias"), contra el PP ("han normalizado y han blanqueado los discursos de extrema derecha") y contra Vox ("fuerza de extrema derecha, postfranquistas sin complejos, una fuerza neoliberal, una fuerza política machista contra las mujeres y contra la gente trabajadora de nuestro país"). Vox atiende especialmente a la corrupción del PSOE y Adelante Andalucía ("36 años de corrupción socialista", "esos andaluces [...] tienen hoy las llaves de San Telmo para expulsar a la corrupción socialista", "ha espantado de Andalucía al comunismo chavista que representa Pablo Iglesias y compañía").

\subsection{Principio de Animacidad}

Este principio está representado por los sentimientos inducidos por los dos bloques ideológicos en cuestiones consideradas vitales para la ciudadanía. Los discursos de la izquierda (PSOE y Adelante Andalucía) apelan al miedo ante la inminente entrada en el gobierno autonómico de la extrema derecha representada por Vox mediante el pacto con PP y Ciudadanos y giran en torno a las consecuencias para la democracia. De esta forma, el PSOE expresa su preocupación y gravedad ("lo preocupante es que una fuerza que se sitúa al margen de los valores constitucionales entra en un parlamento autonómico", "una mala noticia para el sistema democrático en nuestro país"); apunta al riesgo de pérdida democrática y así se manifiesta dirigiéndose a PP y Ciudadanos ("sumar y condicionar la gobernabilidad a la extrema derecha", "su futuro va a ir ligado a la extrema derecha") y culpa al PP de ello ("está dispuesto a cualquier cosa y a sumar sus votos con la extrema derecha para ir contra el Partido Socialista", "la derecha española le ha abierto la puerta de las instituciones a la extrema derecha"). Adelante Andalucía es más drástico en su oposición a Vox y al posible pacto de derechas que ponen en riesgo la democracia con expresiones como: "alerta antifascista", "que no vuelvan, que no vuelvan nunca más", "nos estamos jugando nuestra democracia", "la situación es muy grave", "frente a la extrema derecha, igualdad, libertad y fraternidad".

PP, Ciudadanos y Vox se contraponen al continuismo socialista y a la necesidad de cambio. EI PP reivindica la necesidad de cambio político ante el continuismo del PSOE ("después de 40 años ya era hora de que el Partido Popular gobernara en San Telmo y además lo hacemos con una legitimidad", "les dijimos a los andaluces que había que optar por dos cosas, o por la continuidad o por el cambio", "reivindicar el cambio que ya es necesario en España", "dando la espalda a cualquier acuerdo entre comunistas y socialistas", "romper con cuatro décadas de malas políticas, de casos no muy edificantes, pero, sobre todo, de una resignación que por fin hoy pone punto y final"). 
Para Ciudadanos, el foco se centra en los líderes de PSOE y Podemos ("el sanchismo hoy sale tocado, pero hay que acabar de hundirle en las urnas", "no es gratis pactar con Rufián, con Torra, con Puigdemont", "tampoco le va a salir gratis entregarle la economía a Pablo Iglesias, a Monedero, a Echenique"), mientras que Vox aboga por la necesidad de cambio ("ya estaba bien de entrar en bucle", "se ha acabado el día de la marmota en Andalucía") y se centra en el peligro del separatismo y la desintegración de España por un gobierno del PSOE ("Ios andaluces están muy preocupados con que el golpe de estado separatista haya cambiado de residencia pasando del Palacio de la Generalidad al Palacio de la Moncloa", "los andaluces han dicho aquí donde era más difícil que estén en contra de ese proceso revolucionario que pretenden los actuales socios de gobierno de España").

\subsection{Principio de Rango y Número}

El numeral, las cifras y términos de cantidad, así como los títulos y cuestiones que preocupan a un elevado número de personas o conllevan importantes movimientos también se reflejan en mayor o menor medida en todos los discursos para defensa propia o ataque al contrincante político, pero especialmente, se remarca lo que atañe a muchas personas en número o en relevancia. En el PSOE, Ciudadanos y Vox predominan los resultados cosechados en las elecciones. En el PSOE se repiten estos resultados ("a pesar de [...] haber sido el partido más votado, de haber ganado en 7 de las 8 provincias y haber ganado por 7 puntos al segundo partido y por 10 al tercero"), pero también anota en varias ocasiones la pérdida de votos ("a pesar de ganar hemos bajado en 7 puntos nuestra confianza"). También Ciudadanos concentra los datos en sus propios logros como partido político ("hace cuatro años tenía cero escaños [...] hemos pasado de 9 a 21 escaños", "cuando empezó este proyecto hace once años en Cataluña nos dijeron que nunca iba a ver un cambio, que nunca se podría derrotar al nacionalismo, que nunca se podría derrotar al Partido Socialista en Andalucía y que nunca podríamos gobernar España [...] de esas tres cosas se han cumplido ya dos"). Vox se sirve del principio para destacar los apoyos recibidos en las elecciones ("esos 400.000 andaluces que habéis sido sistemáticamente insultados durante esta campaña", "hace tres años estábamos en Sevilla subidos encima de un banco con un megáfono y nos escuchaban 15 o 20 personas", "permitidme que dé las gracias a esos 400.000 andaluces que hoy nos han respaldado").

En el PP, además de este tipo de datos, resalta sobre los demás por la extensión de la numeración de los logros de los gobiernos anteriores del partido ("este mensaje que hemos llevado por los 9.000 kilómetros recorridos en la campaña, 21.400 desde que soy presidente nacional, en esos 116 actos por Andalucía en 25 jornadas muy intensas ha calado", "después de haberlo hecho en las 8 capitales de provincia, en 5 de las 8 diputaciones provinciales, en 25 de 27 grandes ciudades andaluzas"). Asimismo, presenta su programa inmediato ("hacer funcionar la economía andaluza [...], hacer funcionar los servicios públicos esenciales, esa educación pública y esa sanidad pública, hacer funcionar todo lo que son los instrumentos administrativos") y recuerda su proyecto político pasado, presente y futuro: "que derrotó el Plan Ibarretxe y que derrotó el Plan Puigdemont", "que va a garantizar unas competencias estatales en materia de servicios sociales, sanidad y educación". Este principio también lo utiliza para atacar a la oposición socialista ("el $20 \%$ de los españoles le ha dicho un no rotundo", "no confía ni en sus políticas, no confía en sus aliados independentistas, batasunos, podemitas", "tampoco confían en que pueda representar una esperanza de futuro en materia económica, educativa, en materia de seguridad o en materia también de servicios sociales". 
Y, en Adelante Andalucía la numeración de expresiones abunda en su oposición a la extrema derecha mediante el nombramiento de organizaciones y colectivos diversos de sociedad civil ("un llamamiento al movimiento feminista, a las organizaciones de trabajadores, a las plataformas de afectados por la hipoteca, al movimiento estudiantil, a los colectivos LGTBI, a las organizaciones de pensionistas", "la ciudadanía, los ecologistas y las ecologistas, el feminismo, las organizaciones sindicales vamos a ser capaces entre todos y entre todas de organizarnos mucho mejor", "ante cada palabra de racismo, ante cada palabra de machismo, ante cada palabra de transfobia, ante cada palabra de homofobia nos van a tener delante", "en la calle, en cada barrio, en cada centro de trabajo, en cada pueblo y en cada ciudad la gente de Adelante Andalucía va a estar a disposición hasta hacerle frente a la extrema derecha").

\subsection{Principio de Evaluación Emocional}

Las estimaciones sustentadas en normas culturales son interpretadas por los líderes de los partidos políticos de diferente forma de acuerdo a su ideario, pero en similares proporciones. El PSOE apela en su adjetivación y nominación a los valores constitucionales de democracia, consenso y diálogo y a valores europeístas para defender a España de la extrema derecha ("se sitúa fuera de los valores constitucionales y europeístas", "comprometidos con la democracia, con la Constitución", "próximo a celebrarse el 40 aniversario de la Constitución, sería muy bueno volver a recuperar las señas de consenso", "en otro país europeo esto sería impensable", "en Europa tienen claras dónde están las líneas rojas", "en el resto de los países europeos, este tema estaría resuelto", "tendrán en Europa que comparecer con relación a estas aventuras").

El PP habla del cambio en Andalucía como un reto histórico ("esta bendita tierra a la que tanto amamos, Andalucía, una parte esencial de esa gran nación que es la nación española, necesita como agua de mayo [...] de un nuevo gobierno", "reto histórico de encabezar un gobierno de cambio", "resultado histórico y tremendamente apasionante"), mientras que Ciudadanos expresa su disposición a facilitar gobiernos por el bien de la patria y a plantear alternativas más allá del bipartidismo PSOE-PP ("abandonemos esa maldición bíblica que el Partido Socialista y el Partido Popular nos han hecho creer desde hace cuarenta años", "ha habido cambios y ha habido reformas porque Ciudadanos pensó más en España que en su partido").

Adelante Andalucía llama a la defensa de las conquistas de libertades y derechos de anteriores generaciones ("la España feminista, la España republicana, la España trabajadora, la España democrática debe estar alerta para defender las libertades que nos dejaron nuestros padres y madres, nuestras abuelas y abuelos", "ante la amenaza de acabar con las conquistas sociales que tanto han costado construir en nuestro país, en nuestra tierra, en Andalucía por generaciones precedentes a la nuestra") y Vox aboga por la unidad de España desde la reconquista y la regeneración de valores tradicionales como el centralismo ("programa común que quiere la reconquista, la regeneración de los valores, de esos principios que se han tirado por los suelos", "Vox es sólo un instrumento al servicio de España", "no pedimos papeletas verdes, pedimos papeletas rojigualdas que era lo verdaderamente esencial"). 


\subsection{Principio de Intensidad de la Presentación}

Los líderes políticos han destacado de forma drástica algunas afirmaciones o acciones que piensan realizar con espacios emocionalmente conocidos. EI PSOE apunta al diálogo para contener a la extrema derecha ("abrir el diálogo con todas las fuerzas políticas para intentar evitar y ser dique de contención", "proceso de diálogo entre los demócratas para abandonar la tensión y parar el avance de las fuerzas no constitucionalistas en España", "el combate que nos compete a los socialistas es ahora lidiar el frente de la democracia, la batalla por la democracia frente al miedo"), mientras Adelante Andalucía también apela al acuerdo político ("estamos dispuestos a construir juntos y juntas un dique frente al avance de la extrema derecha", "pedir responsabilidad a todas las fuerzas políticas que sumamos en la mayoría en esa moción de censura"), llama a la esperanza ("queremos trasladar un mensaje de ánimo y de esperanza") y la movilización de la calle para el mismo objetivo ("toca movilizarse para defender las libertades, para defender la justicia social y para defender la fraternidad y, en última instancia, la democracia", "ir a recuperar las plazas, ir a recuperar las calles, ir a ser protagonistas de sus propios cambios"), además de la denuncia ("vamos a denunciar con claridad cada seguidismo que hagan los que se llaman de la derecha formal", "frente a la extrema derecha, vamos a presentar compromiso y antifascismo militante").

EI PP y Ciudadanos hacen el llamamiento a otras fuerzas para llevar a cabo un cambio político. Así el primero, que es el partido que emplea más este principio, se refiere en repetidas ocasiones al cambio ("Andalucía ha votado ese cambio [...] el cambio ha ganado las elecciones en Andalucía", "nosotros proponíamos un cambio, [...], va a haber cambio en Andalucía", "el mandato de los andaluces ha sido nítido", "esta tierra [...] es un fórmula uno; la diferencia es que ahora vamos a poner un buen piloto, con una buena escudería a funcionar el fórmula uno") y aboga por los pactos ("Andalucía no va a tener ningún bloqueo político y hago un llamamiento de manera muy directa a todas esas fuerzas políticas que quieren y desean un cambio", "tenemos tiempo para sentarnos, tiempo para hablar, tiempo para discutir", "abrimos ya la puerta a empezar ya unas negociaciones de cambio, de pactos, que ponga la alternancia necesaria". Ciudadanos expone sus argumentos en la misma línea ("hay diputados que suman para ese cambio, así que el cambio ha llegado a Andalucía", "ese cambio en el que todos creíamos ha llegado") y Vox, por su parte, se hace eco del mandato de los votantes que propician el cambio político histórico ("vamos a propiciar el cambio, la mejoría, la reconquista", "les hemos dicho a los andaluces que era posible y les pedimos una elección histórica", "Ios andaluces una vez más han hecho historia como tantas veces la han hecho a lo largo del tiempo y se han sacudido 36 años de régimen socialista").

\subsection{Principio de Contenido Emocional}

Es usado por todos los todos los líderes políticos, quienes apuntan en algún punto de sus discursos el trabajo de sus colaboradores y de la gente que lo ha apoyado con manifestaciones de agradecimiento y felicitación desde el campo emocional, recreando situaciones de campaña y rememorando sentimientos de ilusión, apoyo, risas, etc. Vox es el partido que más recurre a este principio, donde destaca la búsqueda de la no decepción de los votantes ("reconocer el esfuerzo de todos los que habéis trabajado con nosotros tantísimo", "no vamos a defraudar las expectativas", "os queremos dar las gracias de corazón", "en cada mitin nos hemos encontrado con lo mismo: jóvenes, mayores, trabajadores, gente normal que ha depositado esa confianza y que nos decía no nos defraudéis, pues no les vamos a defraudar", "ha sido una campaña emocionante, ha sido duro, pero ha sido una campaña preciosa porque se nos ha transmitido la ilusión, la confianza, la esperanza de muchos andaluces y españoles que la habían ya perdido". 
Especialmente significativas son las emociones rememoradas por el PP ("yo sé que esas lágrimas que he visto derramarse en las escaleras de nuestra sede o en la propia calle donde estoy viendo a personas llorando después de estos 40 años de socialismo, esas lágrimas son las lágrimas de la esperanza, las lágrimas de la ilusión y las lágrimas del futuro", "esa gran familia se ha puesto a trabajar en esta campaña y lo ha hecho con tesón, con entusiasmo y con mucha ilusión y eso me hace que me sienta muy orgulloso, orgulloso como me siento de Andalucía"). También es demostrativo el orgullo de Ciudadanos ("ha sido un trabajo de equipo", "no sabéis el orgullo que siento, también como hijo y nieto de andaluces", "espero y deseo que hoy Andalucía sea feliz"), así como el ánimo y esperanza de Adelante Andalucía ("queremos agradecer a toda la militancia [...], a todas las personas que han hechos una campaña extraordinaria de defensa de los servicios públicos"). EI PSOE muestra también agradecimiento a sus apoyos en una noche difícil con sentimiento de tristeza ("ésta es una noche triste para los socialistas", "no es una buena noticia que la izquierda haya bajado de esta manera en Andalucía", "han de saber los socialistas andaluces y las socialistas andaluzas que cuentan con todo el apoyo, todo el cariño y el afecto del resto de socialistas españolas y españoles").

\section{Conclusiones}

En primer lugar, resolvemos que, pese a las aportaciones de las neurociencias al estudio de las emociones en el discurso político cada vez más empleado por los políticos, en el análisis científico sus limitaciones tanto en la neuroinformación (restricciones en las fuentes de datos, inestabilidad en el sistema normativo de seguridad y privacidad de datos) como en las técnicas de control cerebral (elevado coste económico y humano, sistema tecnológico muy específico, imposibilidad de análisis comparativos, cuestionamiento de tecnologías de investigación) hacen que los métodos de análisis de la emoción (revisión documental, compendios de estudios, estrategias semióticas, descripción de contextos, repertorios emocionales, análisis multivariante, etc.) más asequibles sean en la actualidad muy variados sin una clara imposición de unos sobre otros.

Por otra parte, hemos comprobado la utilidad de la aplicación de los principios de inferencia emocional de Ungerer al mensaje político televisado desde el análisis descriptivo y de contenido cualitativo de elementos lingüísticos, al margen de los textos en prensa, puesto que hemos observado un amplio uso de estrategias emocionales en los discursos de los líderes políticos, entre las que se encuentran: 1. La evidente dicotomía nosotros versus ellos, siendo el principal eje temático el bloque de izquierdaderecha. 2. Los sentimientos inducidos por los dos bloques ideológicos en cuestiones consideradas vitales para la ciudadanía se circunscriben principalmente en torno al miedo al contrario. 3. Reflejo en mayor o menor medida de cifras y términos de cantidad para defensa propia o ataque al contrincante político. 4. Las estimaciones basadas en normas culturales utilizadas en similares proporciones, aunque de diferente modo según el posicionamiento. 5. Acentuación de afirmaciones o acciones que se piensan realizar drásticamente con espacios emocionales comúnmente conocidos. 6. Énfasis en muestras de agradecimiento y afecto por los equipos de campaña y votantes apelando a sentimientos como la ilusión, apoyo, risas, etc.

Con todo ello, cabe esperar que en un futuro próximo las técnicas de neurociencias salven las limitaciones actuales y puedan ser complementadas con el método de análisis empleado junto a otros observados en trabajos de investigación científica recientes. 


\section{Referencias}

Arroyas Langa, E., \& Pérez Díaz, P. L. (2009). El valor de las emociones en los discursos periodísticos del espacio político. En J. M. de Pablos Coello (coord.), Actas I Congreso Internacional Latina de Comunicación Social. La Laguna, Tenerife: Sociedad Latina de Comunicación Social.

Arteaga Márquez, S. (2018). ¿Cómo funciona el cerebro político? Guía de comunicación política para entender a los votantes y a la opinión pública. Revista Jurídica Mario Alario D’Filippo, 10(20), 187-212.

Cárdenas Ruiz, J. D. (2013). Storytelling y márquetin político: humanidad y emociones en la búsqueda de la visibilidad legitimada. Poliantea, 9(16), 33-50.

Cardona Zuleta, L. M., \& Londoño Álvarez, C. A. (2018). La retórica del miedo como estrategia política. El plebiscito por la paz en Colombia. Forum. Revista Departamento de Ciencia Política, 14, 43-68.

Cebrián Herreros, M. (1983). Fundamentos de la teoría y técnica de la información audiovisual. Madrid: Mezquita.

Charaudeau, P. (2011). Las emociones como efectos de discurso. Versión. Estudios de Comunicación Política, 26, 97-118.

Cossarini, P. (2019). Populismo, acción política y emociones. Líneas de intersección. Anales de la Cátedra Francisco Suárez, 53, 79-95.

Dagatti, M. (2017). Las emociones políticas. Un modelo discursivo de estudio. Rétor, 7(1), 40-72.

Domenach, J. M. (1968). La propaganda política. Buenos Aires: Editorial Universitaria de Buenos Aires.

Eggs, E. (2008). Le pathos dans le discours: exclamation, reproche, ironie. En M. Rinn, Émotions et Discours. L'usage des passions dans la langue (291-320). Rennes: Presses Universitaires.

Espí Hernández, A. (2017). Una propuesta de medición de emociones en las elecciones generales 2016 en España. La convivencia de lo emocional y racional en política que demuestra la supremacía del cerebro límbico. Más Poder Local, 32, 42-50.

Gil, M. (2016). La complejidad de la experiencia emocional humana: emoción animal, biología y cultura en la teoría de las emociones de Martha Nussbaum. Dilemata, 21, 207-225.

Gómez y Patiño, M., \& Bandrés Goldáraz, E. (2014). El neuromarketing: una nueva disciplina para la investigación de audiencias y de la opinión pública. Icono 14, 12, 395-415.

González Quinzán, S. (2019). Emociones y política: el caso de los sentimientos hacia la política en las elecciones gallegas del 2016. Revista de Investigaciones Políticas y Sociológicas RIPS, 18(1), 95-118.

Gravante, T., \& Poma, A. (2018). Manejo emocional y acción colectiva: las emociones en la arena de la lucha política. Estudios Sociológicos de El Colegio de México, 36(108), 595-618.

Gutiérrez-Rubí, A. (2009). La neuropolítica: conocer el cerebro para liderar las ideas. FRC: revista de debat polític, 21, 84-87.

Jiménez Rodríguez, M. (2017). La emoción como estrategia argumentativa en el mitin español. Identificación de actos de habla y análisis cuantitativo. Discurso \& Sociedad, 11(4), 621-641.

López Camargo, S. P. (2018). La construcción de la emoción en los discursos políticos de campaña. Pragmalingüística, 26, 199-220.

Maneiro Crespo, E. (2017). Neurociencia y emociones: nuevas posibilidades en el estudio del comportamiento político. Revista de Investigaciones Políticas y Sociológicas RIPS, 16(1), 169-188.

Medina Delgado, W. A. (2015). Apelaciones a la emoción en la argumentación. Folios de Humanidades y Pedagogía, 3, 105-126.

Méndez Muros, S. (2014). La imagen emocional de Latinoamérica en la prensa digital española: Elpais.com como estudio de casos. F@ro, 19(1), 93-112. 
Mendieta Ramírez, A. (2018). Cerebro político en los procesos electorales: las emociones del voto en la campaña por la Presidencia de la República en México, 2018. Anagramas Rumbos y Sentidos de la Comunicación, 17(34), 45-69.

Nocetti, O. R. (1990). Falacias y medios de comunicación (el discurso como arma). Buenos Aires: Humanitas.

Perelman, C., \& Olbrechts-Tyteca, L. (1989). Tratado de la argumentación. La nueva retórica. Madrid: Gredos.

Sierra, M. (2015). Entre emociones y política: la historia cruzada de la virilidad romántica. Rúbrica Contemporánea, 4(7), 11-25.

Tallón-Ballesteros, A. J. (2019). Metaheuristic algorithm to train product and sigmoid neural network classifiers. Expert Systems, 36(1), e12383.

Tapia Frade, A., Matellanes Lazo, M., Berdón Prieto, P., \& Martín Guerra, E. (2016). Neurociencia en comunicación política: un experimento sobre los discursos políticos en España. Opción, $79,50-66$

Ungerer, F. (1997). Emotions and emotional language in English and German news stories. En S. Niemeier \& R. Dirven (Eds.), The Language of Emotions. Conceptualization, expression, and theoretical foundation (307-328). Ámsterdam/Filadelfia: John Benjamin Publishing Company.

Valdez Zepeda, A., Huerta Franco, D. A., \& Díaz González, S. (2014). La vergüenza y el orgullo en la política electoral. El caso del sistema de partidos políticos y los medios de comunicación en México. Revista Enfoques, 12(20), 115-132.

Van Dijk, T. A. (2006). Discurso y manipulación: discusión teórica y algunas aplicaciones. Revista Signos, 39(60), 49-74. 